\title{
Real time US -guided ventricular catheterisation: a single centre retrospective audit
}

\author{
Erjon Agushi, James Montgomery Barber, Konstantina Karabatsou \\ From Hydrocephalus 2015 \\ Banff, Canada. 18-21 September 2015
}

\section{Introduction}

Suboptimal shunt or external ventricular device (EVD) catheter placement can result in significant morbidity, requiring system revision and consequently prolonging inhospital stay. Multiple incorrect trajectories can potentially cause further complications and neurological deficits. While experienced operators can achieve relatively high success rates with the craniometric technique, the failure rates can be even greater than $20 \%$, in certain studies.

\section{Design}

Retrospective audit of documented method of guidance technique and subsequent clinical/radiological outcomes.

\section{Objective}

The objective of our study was to compare the clinical benefits of real-time Ultrasound guidance in enhancing ventricular catheter insertion versus the standard craniometric-guided free-hand technique.

\section{Methods}

Operative records, clinical outcomes and postoperative scans of the patients who underwent EVD or shunt insertion from between March 2013 to March 2014 at a single centre were reviewed retrospectively.

\section{Results}

A total of 162 patients' clinical notes and scans were reviewed. 22 were excluded due to unclear documentation, missing operative notes or postoperative scan having not been performed. 78 underwent an EVD insertion, mainly for secondary hydrocephalus due to SAH, whereas 62 had insertion of a VP shunt as a primary procedure. Use of US to visualize the ventricles was documented in $57.9 \%$ (56.4\% in EVDs vs. 59.7\% in shunts), while usage of

* Correspondence: erjon.agushi@srft.nhs.uk

Salford Royal NHS Foundation Trust, UK 